\title{
Reliability and Safety Aspect of Active Magnetic Bearings Applications
}

\author{
Prince Owusu-Ansah ${ }^{1}$,Philip Kwabena Agyeman ${ }^{2}$, Timothy Alhassan, ${ }^{3}$ \\ Saviour Kwame Woangbah. \\ ${ }^{1}$ Mechanical Engineering Department, Kumasi Polytechnic P. O. Box 854, Kumasi Ghana, West Africa. \\ ${ }^{1}$ School of Mechanical and Electronic Engineering, Wuhan University of Technology,P.R China, 430070 \\ ${ }^{2}$ School of Mechanical and Electronic Engineering, Wuhan University of Technology,P.R China, 430070 \\ ${ }^{2}$ Mechanical Engineering Department, College of Engineering, KNUST, Kumasi Ghana, West Africa. \\ ${ }^{3,4}$ Mechanical Engineering Department, Kumasi Polytechnic P. O. Box 854, Kumasi Ghana.West, Africa.
}

\begin{abstract}
This research work discusses reliabilityand safety aspectof active magnetic bearings applications in high industrial rotational machinery. A structure of the smart machine presented in this research work demonstrates a schematic method of improving industrial machinery performance by the use of active magnetic control technology. The smart machine consists of a tool of three main parts, the actual mechatronics system, the real machine with processor sensor, the actuators and thecontroller. In this study the rotor is used as a machine tool for the magnetic bearing.The active magnetic bearing is said to be a typical example of a mechatronic device which consist of mechanical, electrical as well as information processing systems and elements. Defects disturbances associated with microprocessor hardwaresuch as, breakdown of mechanical system components, blade loss, rotor crack, as well as cooling system leakageswhich are usually present in the power supply systemhave all been studied and discussed.
\end{abstract}

Keywords: Actuators, Active magnetic bearing, Control, Safety Sensors, Reliability.

\section{Introduction}

The use of active magnetic bearings for the suspension of high speed rotors in industrial machinery has gain dominance in recent times and has become the state of the art component ranging from the area of research through to the prototype design stages, to the area of industrial application such as small turbo-molecular pumps to high power pipeline compressors measuring in the ranges of megawatts power. Industrial users and players in these field are of the known that, beyond the basic functions of this machinery,reliability andsafety aspect of this equipment's is very critical to its continuous commercial use and development.

Safety is more than a mere technical use. It involves a strong aspect of psychological interpretation, and societal demands, and so safety aspect in high industrial machinery should be of outmost concern. Reliability on the other hand has a definite and technical sense of touch, and it appears to be of more importance to engineering calculations and of economic considerations.

Mathematical tools are available for the purpose of assessing reliability of classical technical system and performance numbers for the purpose of comparison in situations such as the mean time between failures. Reliability analysis in a given technical structure and system consist of a more or less large number of classical components which have all been well developed in [1], However it is while noting that active magnetic is not a classic case of a technical system, it is of a more typical mechatronic product, hence it contains an information processing system, software and feedback control loops.

In addition to the synthesis of a safe mechatronic system, the method of designing it, is not obstructed in a systematic way, there is however a strong opportunity to make use of a Mechatronic system, despite its complexity which are said to be more reliable than the classical ones [1].

The potential of having an internal processing information system, which have the ability to resemble living beings uses information to increase the "chances of survival" could make mechatronics system more reliable.

\section{Literature Review}

Safety is usually seen as an ambiguous term, and usually is off importance to consider the nontechnical side as well. Danger has always been considered as an integral part of human life, and the absence of safety could result in damage of properties and loss of precious human life. Danger may occur from environmental catastrophe wild animals, unknown enemies or unexpected illness. The society in which we live on, must all take consensus effort to covert environmental danger into risk, in order to make it calculable, predictable and controllable, to tame fate. The construction of hydro dam has been built to avoid possible 
flooding.The approach of technical means to increase safety in many advanced products, are mainly based on the concept of Mechatronics [2]. The driving of automobile cars has been made safer as drivers are able to assist systems which control dangerous situations, such as braking or skidding.

The acceptance level of danger and risk has a very strong psychological background which varies with emotional attitude, habits and individual exposure. In hobby sports, people normally enjoy the thrills of risk, but bungee jumping, or car racing are typical example of line initiative discussion of Raymond Mays, a participant of a car race near Cardiff in 1924 lost one of his wheels while driving with a speed of more than $90 \mathrm{~km} / \mathrm{h}$ is shown in Fig .1.

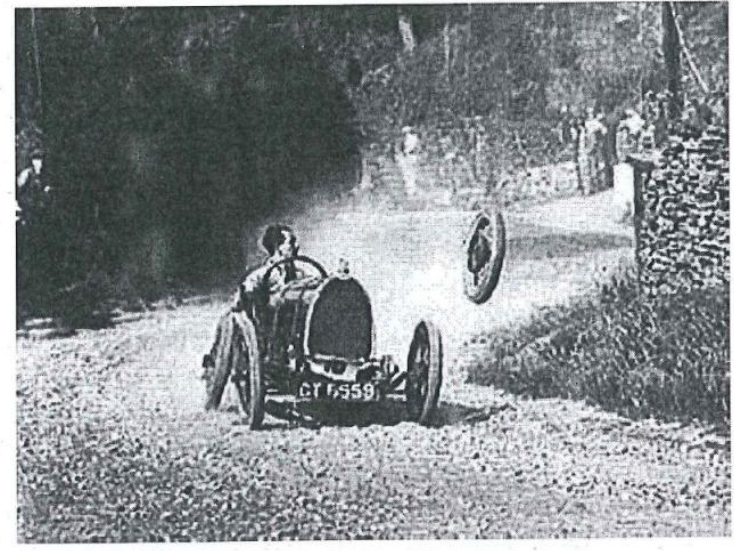

Fig. 1.Raymond Mays, a participant of a car race [1]

With the issue of philosophical, safety might spur some discussion as well. The philosopher Karl Popper [2], in his famous treatise Logic of Science, 1934 did asserted that any progress in Science probably will be as a result of falsifying the existing theories and eventually modifying them with new ones that corresponds to better experience than the previous one. This then means that you cannot prove that a theory is right, but you can only try to falsify it or part of it and then eventually improve the situation. In consequence, a statement that states a system is safe, describe an ideal state that cannot be verified, but only to some extent can be falsified. It is an uncomfortable insight to many people that risk is a situation that principally, cannot be avoided. However, there are various techniques to reduce risk.

\section{Technical Aspect of Safety, Reliability And Dependability}

Safety is seen as one of the four aspect of dependability, a term coined by Laprie in 1992. Dependability comprises safety, reliability, availability and security [3]. Availability is defined as the readiness for usages; Security addresses the access of a system, the authority to operate such as issuing commands and altering the software system.

In summary security consist of regulation for the communication to the outside world from the technical system under consideration [4].

The terms safety and reliability somehow overlaps and a typical example been illustrated in Fig.2.

Safety is the quality of a unit to
represent no danger to humans
nor environment when the unit
fails (technical safety). It is
investigated by reliability theory.

Safety is the quality of a unit to nor environment when the unit investigated by reliability theory.

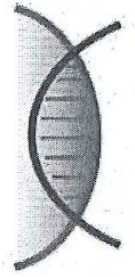

Reliability is the quality of a unit to remain operational. It characterizes the probability to have no interruption of operation during a certain time.

Fig. 2. Relationshipbetween Safety and Reliability

Engineers and designers are more interested in this overlaps, as result they are able to attain a safe and a more reliable product. In the foregoing section the active magnetic bearing component and its safety aspect have been discussed.

\section{AMB As A Mechatronic Product And A Failure Example}

An active magnetic bearing is said to be typical example of a mechatronic device which consist of mechanical, electrical and information processing element. An application area of such a mechatronic product 
which is been well established and developed is the turbo-machinery unit, this product ranges from small turbomolecular pumps, large compressors, turbo - generator for power plant as well as pipeline gas layouts. Examples of system software failure which are associated in a typical AMB as a Mechatronic product are system breakdown, run- time exception such as address errors and bus time -out, up to incompatible program version.

When electronic systems fails, or the signal processing unit get obstructed or disturbed which are more often due to excessive noise oriented from the electromagnetic source, this situation are usually mistaken as a sensor signal.

The area of EMC (Electromagnetic Compatibility) is usually taken seriously, taking into consideration the high-power switch amplifier in the AMB loop.

Defects associated with microprocessor hardware anddisturbances which are normally present in the power supply have been taken into accounts, for such mechanical failures, the breakdown of mechanical parts, such as the blade loss, rotor crack, or leakages in the cooling system are failure modes which are well known from classical rotor design. The major sources of failure which are worth mentioning are excessive mechanical load as well as a high centrifugal force at high rotor, usually this limitation is attributed to as a result of the strength of the material of the rotor, the magnetic bearing area and the design of motor part.

\section{Measures of Reducing Risk of Failure}

Different types of measures associated in reducing the risk offailure within a Mechatronic product ranges from systematic design procedures, software development tools, individual measures, redundancies and quality control to smart machine concept that included various control strategies, active fault diagnostics and corrections, have all been discussed in the subsequent subsection.

\subsection{Quality Control Standards}

In quality control approach a systematic approach which introduces quality aspect in the production design and the operation of its product and systems uses standardized procedures as contained in ISO 9000 series [5], which has been accepted worldwide. The field of AMB research is still young whiles the study of evaluation of vibration in AMB are under development by ISO. The America Petroleum Institute(API) for example consists of an informative section on application consideration for active magnetic bearing to its API Standard which are very useful in the applications of active magnetic bearing [5].

\subsection{Systematic For A Product Design}

Classical methods of ensuring best practicing of the state of the art is to employ the use of Failure Modes Effect and Critical Analysis(FMECA) approach for monitoring and checking active magnetic bearing designs. In such approach a group of expert with different knowledge and expertise, ranging from design, production, repair test, and potential users are made to evaluate the product and the design. These experts are made to identify potential failures modes, determine the effects and the consequences of such failures and to suggest various modifications of the design with the aim of improving its efficiency.

\subsection{Software Development}

In mechatronic product development, the software system is as an integral part of the product: In such situation, the software is developed and implemented, of course, the software must be logically correct and the operating system then takes care of the syntax. But, in such instances the correct time sequences of the computational task is very essential in real time applications.

In the application of industrial AMB, proprietary software is most often run on single clip digital signal processors(DSP) giving an efficient and economic solution. Software for such applications are usually streamlined and dedicated to specific task with well-defined constraints.

In the cases where experimental set-up approach are employed, the task are usual much diverse and more complex which requires versatile approach and solutions. In situations of complex task, it may not be sufficient to just use high - speed computers involving high sampling frequency with the hope that this will be adequate for real time operation. In such situation it might be suitable to use a real time operating system(RTOS) from the onset in order to be able to develop and finally operate the software, such RTOS are readily available in various versions, such as RT Linus, dSPACE, AND VxWorks, mostly overhead size, speed and availability.

The design of the software is still an art and ongoing design process, but nevertheless, there are a number of accepted methods for designing and validating complex software design.

One of the methods employed for reducing the probability of errors that are associated with such software design development system is illustrated in Fig.3. 


\section{DESIGNER}

$$
\text { interactive user interface on a PC }
$$

\begin{tabular}{|c|c|c|}
\hline $\begin{array}{l}\text { interactive } \\
\text { configurator }\end{array}$ & $\begin{array}{c}\text { standard } \\
\text { library }\end{array}$ & $\begin{array}{c}\text { user } \\
\text { library }\end{array}$ \\
\hline simulation & emulation & $\begin{array}{c}\text { test and } \\
\text { operation }\end{array}$ \\
\hline
\end{tabular}

\section{PROCESS}

Fig.3. Basic Concept for a Software Development System for Embedded Microprocessor

The design makes use of software packages configuring them interactively with graphical tools. The RTOS adopted is the same as the design and the process application, which allows for meaningful simulation and emulation, fast modifications and realistic test. This software packages which are used for rapid control prototyping are usually very versatile and useful for the design of embedded systems, these includes signal processing tools and actuator drivers which allows for hardware-in loop test[6].

In addition, a development system which consist of extensive modelling of the rotor in AMB facilitates the simulation of various design variation which is aim at optimizing its safety features.

\section{Redundancy}

Another way of improving reliability of active magnetic bearing applications is to use redundant components and information to perform this action. There are two different kinds of redundancies, namely hardware and analytical redundancy. When a failure of a single component cannot be corrected and is deem critical to the systems safety, the function of the component have to be arranged in parallel, in order to replace the failed component as shown in Fig.4i. In such situation appropriate failure detection and switchover schemes are very critical, and the increase in the numbers of the components counteract the overall reliability.

In another situation, if the function of a component is at least partially performed by another component then the functional relation between these existing components can be used as an analytical redundancy for replacing the failed component partially, or for the purpose of reducing the extent and the cost of the hardware redundancy as shown in Fig .4ii
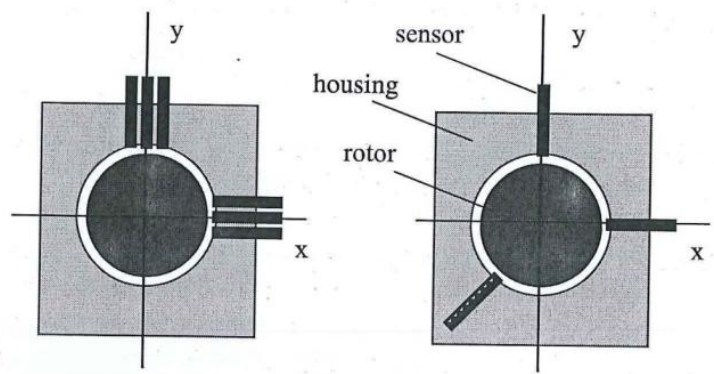

(i)

(ii)

Fig. .4. Cross- Section of a Rotor/Bearing with Redundant Sensors. (i) Hard ware Redundancy with Triplex Sensors Configuration, (ii) Analytical redundancy, Simplex sensor Configuration with one Redundant Sensor

\section{Robust Control}

The design of robust control for AMB must allow for operational uncertainties in the system parameter and for variety of disturbances acting as additional inputs to the sensors due to the fact that classical control can no longer be handled because of theuncertainties which may arise in the bearing characteristics due to temperature rise. The rotor mass which is modified by its inertia of its gas being transported in turbo-machine, or the damping characteristics of the flexible rotor. These disturbances acting as strong additional inputs are most often than not from the external source of the system. These disturbances arise from the motion of the machine based which are caused by earthquakes or as a result of using the AMB/Rotor system in a moving vehicle, or from a tool-generated forces in a milling process as robust control operations often requires high order controller modulation. 


\section{Fail Safe System of an Auxiliary Bearing}

The best way to build a safe system is to make the system itself fail/safe. This laterally means if anything goes wrong, then as a last resort, the system will have to degenerate to a safe system. An aircraft is an example which is not a fail/safe system. For this reasons AMB systems are equipped with auxiliary bearings. Auxiliary bearing are set of additional bearings in which the rotor is made to come into contact only with the auxiliary bearings when the contact free suspension with AMB is not functioning, fails, or operates under heavy varying load.

An example of an active auxiliary bearing design is a special design ball bearing or a simple retainer bearing which are used for touch down bearings. In such situation where the friction between the spinning rotor and the touch down bearings are too high, a violent destructive back whirl is usually developed and noticed. During this whirl the contact force becomes quite high up to about 300times the rotor weight, the touch-down bearing of the rotor can be a serious safety hazards, as such the study and the understanding of touch - down dynamics and the design of the bearings are considered as an important aspect in AMB designs.

\section{Smart Machine Technologies}

In mechatronics, the basic idea is combining mechanics, electronics and information processing within a product in a synergetic way which is usually used to develop the concept of smart machines in which the capability of the internal information processing is used in an extensive way.

Technologies employed in this design ensures that these smart machines and is able to identify its internal state and to be able to optimize itsinternal information processing at the same time. This ensures better functionality with features such as self- diagnostic which eventually leads to less maintenance and higher safety.A block diagram illustrating a structure of a smartmachine in Fig.5demonstrates the schematic method of improving machinery performance by the use of active magnetic control technology.

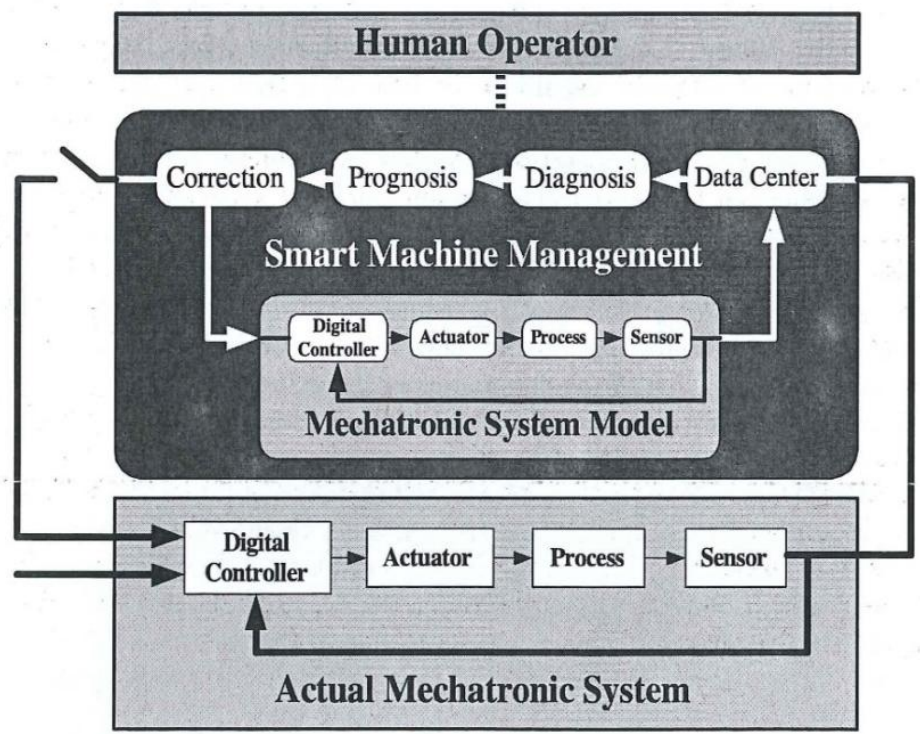

Fig. 5. Structure of a Smart Machine

The smart machine illustrated in Fig. 5 consists of a tool of three main parts, the actual mechatronics system, the real machine with processor sensors and actuators and a controller. The rotor is used as a machine tool or a turbo rotor for the magnetic bearing. The second part is the mechatronic system model in which a software represents the real machine, as such setting up such a model may not be simple as such identification techniques have been considered as an important tool in this technology.

The model at the end will be used for designing, modifying and reconfiguring the control of the real machine. The third part describes the smart management which indicates the additional functioning that are incorporated into the system by making "smart" the use of available information. At first instant, data was collected from the real machine, its sensors, as well as gathering data from the model, which runs parallel to the real machine. On the basis on this information available a diagnosis of the present state is possible and then finally predicted. Furthermore, due to the in built features in the control loop, self-diagnosis and active diagnosis is possible, that is the system itself is able to derive hypotheses about parameters or faults and to check the system by creating suitable test signals for the model and for the real system. This approach usually 
improvesidentification procedures and it is of great interest for reliability management, in that the system itself could be able to identify failures in mechanical components such as crack in the rotor or the location of excessive unbalances, or failures in electrical component such as the sensors.

Identification techniques have been extensively developed for multivariable AMB system[7]. The procedure is to first identify the structure of the unstable open system during close loop operation, the result is then extended and used to derive an automated, iterative way of robust controller for a flexible rotor.

An experimental set-up shown in Fig .6and Fig.7 represents the realization of the structural block diagram in Fig .5. In addition to the control loop for supporting the rotor, a diagnosis and a correction module has been implemented.

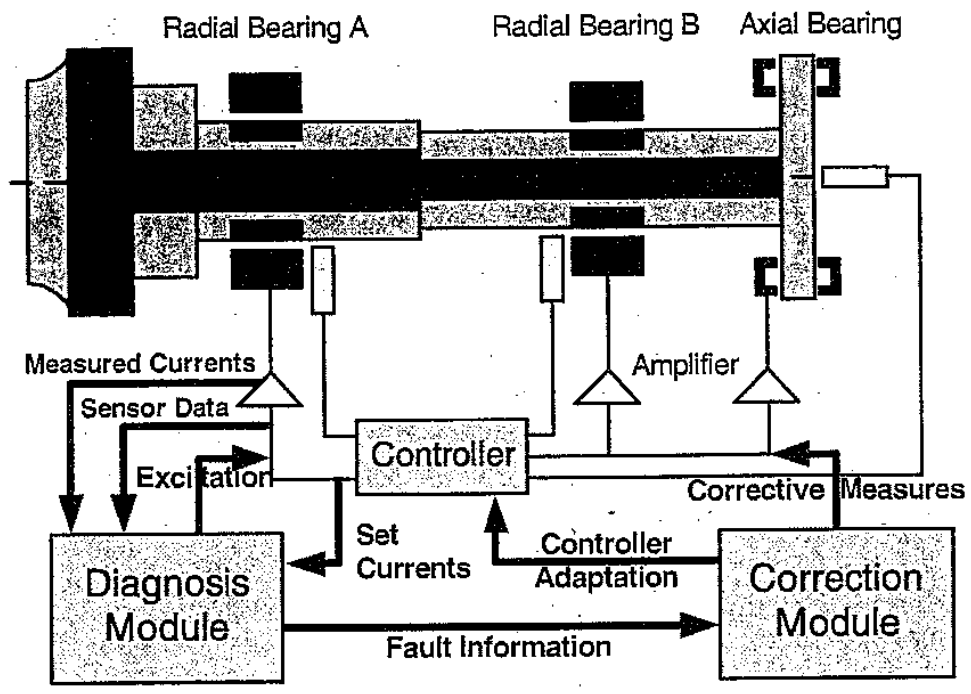

Fig. 6. Rotor in AMB with Additional Smart Machines Modules for Diagnosis and Corrections

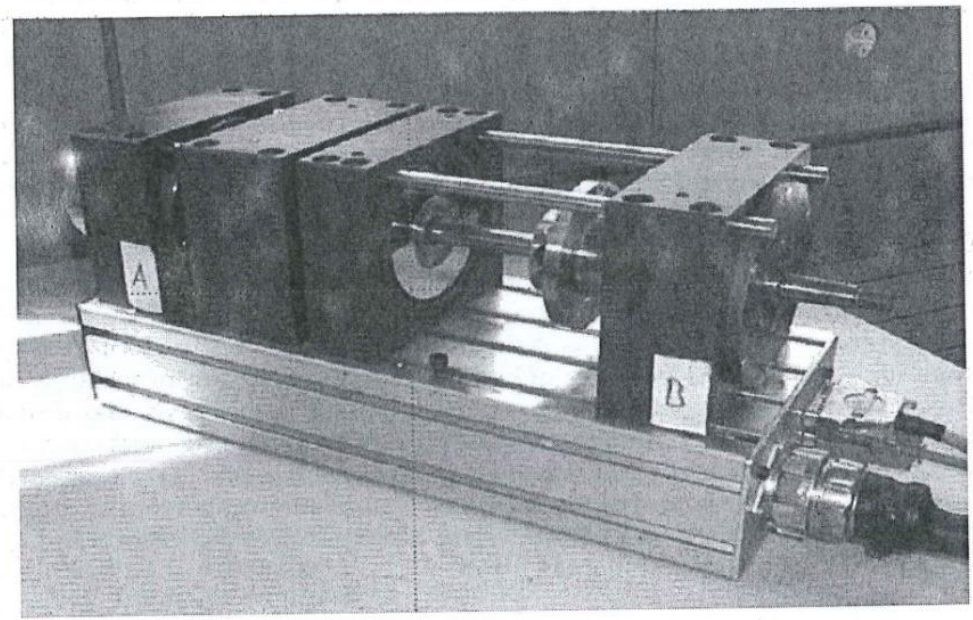

Fig.7. Test Rig for Smart Machine Technology

Identification diagnostics tools have well been developed and are being used for fault detection of various kinds and for the development of fault -tolerant control. In magnetic bearings, faults in sensors and actuators have been detected and corrected using identification procedures. The techniques used spans from processing the bearing sensors signals appropriately up to the use of sophisticated identification software procedures from the level of monitoring the deviation of normal operating behavior to actively switching to an alternatively control method of strategy in case of need.

A typical example of such situation is the malfunction and the wear of tools in milling processing machines which can be diagnosis from the signal of the AMB- supported milling spindle. It can also be said that AMB can be used to detect faulty process parameter which are not directly related to the functioning of the AMB itself and in such situations the AMB can also contribute to the reliability of the whole machine and its working process. 


\section{Conclusion}

The term safety as used to describe a product is an ambiguous word that certainly requires more consideration from the user view point. Philosophical reasoning on the logic of science shows that safety measures can be improved, when is considered step by step but it should be noted that it cannot be guaranteed.

In this view the main emphasis is usually on the technical aspect, its safety and reliability of AMB/ rotor systems. Various methods mechanicalfailures that are associated with mechatronic systems with special emphasis in AMB have been studied and discussed.

In this research reliability and safety have been put into the framework of quality management and design, and then specific ways of dealing with active magnetic bearing have been considered. Redundancy schemes, various exception handling schemes and robust control are all proven tools which have been discussed and numerous examples cited in this study.

The potential of active magnetic bearing becoming fault-tolerant is seen as a general and a unique feature of smart machinery. Smart as used in the study refers to a machine that knows its state since it already has sensors which consist of internal control loops for its functionality which makes best use of the internal information processing capabilities within the machine to be able to optimize its state.

The design of fault-tolerant AMB using diagnostics, identification methods and reconfigurable control features have been discussed in this work. It is expected that future research work will focus on the trends of identification procedures and make them available for further applications which could contribute to the already very impressive, but still growing area of reliability and safety features that needed and are associated with the design and operations of AMB applications.

\section{Acknowledgements}

This work was supported by The Natural Science Foundation of China (NO.51275372) and Wuhan High-Tech Development Project Foundation (NO.201110921299) and The Fundamental Research Funds for the Central Universities (Wuhan University of Technology No.2012-IV-036).

\section{References}

[1]. H. Pham, editor Handbook of reliability Engineering. Springer-Verlag, 2003, 503-509.

[2]. K.R. PopperLogik der Forschung. Springer-Verlag, Wien, 2008, 452-501.

[3]. J.C. Laprie.Dependability: Basica Concepts and Terminology. In Proc. IFIP, on Dependable Computing and Fault Tolerance 23(5) 1992, 234-240

[4]. Koopman. H. Madeira. Dependability Benchmarking and predicting: A Gradn Challenge Technology Problem/ In the Proc. Ist International Workshop o Real Time Mission -Critical Systems. 9(7) 1999, 505-508.

[5]. D. Diez and G. Schweitzer. Simulation Test and DiagnosticIntegrated for the Safety Design of Magnetic Bearing Prototypes. In G. Schweitzer., M. Mansour, Editors, Proc. IUTAM-Symp. On Dynamics of Controlled Mechanical System, 15 (8), $2009,501-510$.

[6]. D. Ewins., R. Norman., G. Schweitzer., A. Traxler. Improved Machinery Performance Using Active Control Technology. Tenth International Symp. On Magnetic Bearings, 15(20), 2008, 149-157.

[7]. Shiyu, Z. (2001). Active Balancing and Vibration Control of Rotating Machinery, Fifth International Conference of Survey, Shock and Vibrational Digest, 33(5), 361-371.

[8]. L. Guoxin, M. Eric, and A. Paul, A note on ISO AMB- Rotor System Stability Margin, In proceedings of the Tenth International Symposium on Magnetic Bearings, 14(16), 2006,156-167. 